\title{
A local controllability test for nonlinear multiple model systems
}

\author{
Todd Murphey, Joel Burdick \\ Engineering and Applied Science, California Institute of Technology \\ Mail Code 104-44, Pasadena, CA 91125 USA \\ \{murphey, jwb\}@robotics.cal tech.edu
}

\begin{abstract}
This paper develops a local controllability result for Multiple Model Driftless Affine (MMDA) control systems. The controllability result can be interpreted as a non-smooth extension of Chow's theorem, and uses a set-valued Lie Bracket. These results are interpreted in terms of an illustrative example involving an overconstrained wheeled vehicle.
\end{abstract}

\section{Introduction}

This paper considers the issue of controllability for systems of the following form:

Definition 1.1 A system is said to be a multiple model driftless affine system (MMDA) if it can be expressed in the form

$$
\dot{q}=f_{1}(q) u_{1}+f_{2}(q) u_{2}+\cdots+f_{n}(q) u_{n}
$$

where for any $q$ and $t, f_{i} \in\left\{g_{\alpha_{i}} \mid \alpha_{i} \in I_{i}\right\}$, with $I_{i}$ an index set, $f_{i}$ measurable in $(q, t), g_{i}$ analytic in $(q, t)$ for all $i$, and the controls $u_{i} \in \mathbb{R}$ are piecewise constant and bounded for all $i$.

Among other things, this implies the control vector fields may change, or switch, amongst a finite collection of vector fields, each representing a model, $P$, in a set of models $\mathcal{P}$. In the case studied in this paper, the switches between models are determined by a collection of $\mathcal{C}^{1}$ continuous submanifolds $\left\{N_{k}\right\} \subset \mathbb{R}^{n}, k=1, \ldots, p$. Fig. 1 depicts the state space of a simple example where two different regions, A and $\mathrm{B}$, correspond to different governing equations. The region boundaries are denoted by $N_{1}$ and $N_{2}$. Within each region, the governing model is unique. As the system trajectory flows from one region to the other, its governing equations switch at the boundary. The difficulty addressed in this paper lies in the fact that the regions' geometries may be a priori completely unknown, and moreover may be local in naturei.e. Fig. 1 may correspond to an arbitrarily small neighborhood of the operating point. Moreover, we allow the $\left\{N_{k}\right\}$ to be unknown $C^{1}$ submanifolds of $\mathbb{R}^{n}$ with an arbitrary, but finite, number of intersections between submanifolds.

Such systems are intimately related to multiple model systems such as studied in Hespanha et al. [1999] and Murphey and Burdick [2001]. However, we should emphasize that the "switching" which occurs when the trajectory $q(t)$ crosses state space boundaries is not like the switching

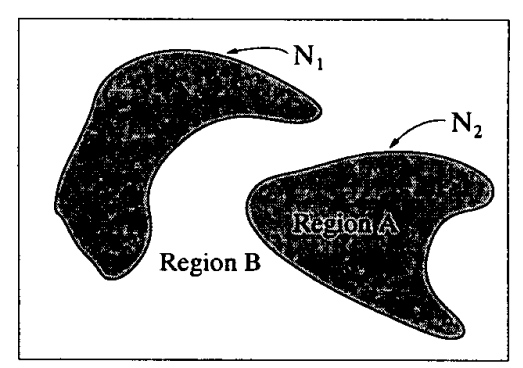

Figure 1: Schematic depiction of state space

phenomena found in Branicky [1998], Liberzon and Morse [1999], Dayawansa and Martin [1999], or Zefran and Burdick [1998], or as typically studied in the hybrid control systems literature (e.g., Pappas et al. [2000], Asarin et al. [2000]). In these studies, the switching is part of a control strategy to be implemented in the controller. Rather, it is switching induced by environmental factors, such as variations in the contact state between rigid bodies. Systems of this sort are actually quite common in engineering practice (see Section 4 for an example). As a first step in understanding such systems, we would like a local controllability test that works in the presence of a priori unknown switching behavior. This paper presents a "discontinuous" version of the Chow's theorem that extends the classical result in a natural way to the systems of Def 1.1.

The issue of controllability for such systems has not been extensively addressed. While controllability was not studied by Hespanha et al. [1999], they did consider a related stabilization problem arising from a kinematic nonholonomic vehicle with parametric uncertainty. In Murphey and Burdick [2000] and Murphey and Burdick [2001] we considered the local controllability problem of multiple model systems, but required relatively strong assumptions on the type of switching that occurred. Goodwine and Burdick [2000] developed a local controllability test for systems of the form in Definition 1.1 when the switching boundaries and configuration space have an a priori known stratified structure. While they did not study multi-model systems, Rampazzo and Sussman [2001] have recently developed a nonsmooth version of Chow's theorem that applies to Lipschitz vector fields. The results obtained by Rampazzo and Sussman [2001] have strong analogues with our result. While the focus of this work is not 
adaptive control, note that multiple model plants can arise in the context of adaptive control schemes where a system is known to have the dynamics of one plant $P$, chosen out of some set $\mathcal{P}$, and one wishes to control the system knowing only properties of the set $\mathcal{P}$. See, e.g., Hespanha et al. [2001].

As an example of a physical system where these concepts are important, Section 4 analyzes a simple model of an overconstrained wheeled vehicle, which is inspired by novel high-mobility wheeled robots (e.g, the Mars Sojourner) that operate in rough terrain. For more details on these vehicles and their control issues, see Murphey and Burdick [2001].

\section{Background}

The primary goal of this paper is to extend Chow's Theorem (reviewed in Appendix A) to the MMDAs of Definition 1.1. We use several aspects of the formalism of Filippov [1988] for investigating the properties of ODEs with discontinuous right hand sides. Eq. (1.1) can be viewed as a differential inclusion, i.e., a system of the form $\dot{q} \in F$, where $F$ is a set valued multi-function. For equations of the form $\dot{q}=f(q)$ with $f$ discontinuous in $q$ at a point $q_{*}$, one must generally allow $f$ to take on the convex hull of limit values $\lim _{q \rightarrow q_{*}} f$ at $q_{*}$ in order to guarantee existence of solutions (see [Filippov, 1988, Chapter 2] for details). To account for this issue at the switching boundaries, we define the following at each $q$ :

$$
\gamma_{i}(q)=\operatorname{co}\left\{f_{i}(q)\right\}=\operatorname{co}\left\{g_{\alpha_{i}}(q) \mid \alpha_{i} \in I_{i}(q)\right\} .
$$

where $I_{i}(q)$ is the set of limiting values of $f_{i}(q)$ at $q$, and $c o\{\cdot\}$ denote the convex hull of a set. For notational convenience, let $s\left(\gamma_{i}\right)$ denote a selection of $\gamma_{i}(q)$-i.e., a choice of a particular vector from $\gamma_{i}(q)$. Let $S_{i_{1}, i_{2}, \ldots, i_{k}}$ denote the set of all possible selections from $\gamma_{i_{1}}, \ldots, \gamma_{i_{k}}$.

Our result uses the notion of a set valued Lie bracket. This concept has its origin in two distinct areas. Previously in Murphey and Burdick [2000] we used a set-valued Lie bracket to consider the local controllability of MMDAs in the special case where switching occurs very rapidly. The use of a set-valued bracket was a natural consequence of the underlying assumptions in Murphey and Burdick [2001]. Rampazzo and Sussman [2001] use a set-valued Lie bracket to prove the controllability of a driftless affine control system whose single governing equation includes Lipschitz control vector fields. They showed that this choice of Lie bracket is a General Differential Quotient of the product of exponentials formulation of a Lie bracket. Although these two applications seem different, the choice of Lie bracket is the same, and the resulting non-smooth versions of Chow's theorem are analogous. Rampazzo and Sussman [2001] use the following Lie bracket definition, adapted here to our situation:

Definition 2.1 Let $f_{1}$ and $f_{2}$ be as in Def 1.1. 1.e., $f_{i} \in$ $\left\{g_{\alpha_{i}} \mid \alpha_{i} \in I_{i}(q)\right\}$. The Lie bracket of $f_{1}$ and $f_{2}$ is defined as

$\left[f_{1}, f_{2}\right](q)=c o\left\{\lim _{j \rightarrow \infty}\left(D f_{1}\left(q_{j}\right) \cdot f_{2}\left(q_{j}\right)-D f_{2}\left(q_{j}\right) \cdot f_{1}\left(q_{j}\right)\right)\right\}$ for all sequences $\left\{q_{j}\right\}_{j \in \mathbb{N}}$ such that

1. $f_{1}$ and $f_{2}$ are differentiable $\forall q_{j}$,

2. $\lim _{j \rightarrow \infty} q_{j}=q$,

3. the limit of (2.2) exists.

Note that this Lie bracket is a set valued object, which can be shown to be both compact and convex. Definition 2.1 is appropriate to the case where the dynamics are single valued in open neighborhoods, but multi-valued on "switching boundaries." In the case where $f=c o\left\{f_{i}\right\}$ and $g=c o\left\{g_{j}\right\}$ on the boundary submanifold $N_{k}$, it is straightforward to show that $[f, g]=\operatorname{co}\left\{\left[f_{i}, g_{j}\right]\right\}$. Definition 2.1 is equivalent to a set-valued bracket defined previously in Murphey and Burdick [2000], where it was used to show local controllability for MMDA systems undergoing rapid switching.

To analyze the controllability of MMDAs, we define:

Definition 2.2 Let $f_{i}$ be as in Def 1.1 and $\gamma_{i}$ as in Eq. (2.1). Define a distribution $\Delta_{s_{1} s_{2} \cdots s_{n}}(q)$ as

$$
\Delta_{s_{1} s_{2} \cdots s_{n}}(q)=\operatorname{span}\left\{v \mid v=s_{i}\left(\gamma_{i}(q)\right), \quad i=1, \ldots, n\right\}
$$

That is, $\Delta_{s_{1} s_{2} \cdots s_{n}}(q)$ is formed from a particular selection of vectors from each $\gamma_{i}(q)$. Define the distribution $\Delta(q)$ as:

$$
\Delta(q)=\bigcap_{S_{1, \ldots, n}} \Delta_{s_{i} s_{2} \cdots s_{n}}(q)
$$

That is, $\Delta(q)$ is formed by intersecting the $\Delta_{s_{i} s_{2} \cdots s_{n}}(q)$ over all possible selections of $\gamma_{1}(q), \ldots, \gamma_{n}(q)$. Next define

$$
\Delta^{1}(q)=\bigcap_{s_{12,13, \ldots}}\left(\operatorname{span}\left\{v \mid v=s_{i j}\left(\left[f_{i}, f_{j}\right]\right)\right\}\right)
$$

and analogous higher order distributions formed from higher order set-valued Lie brackets. Finally, define $\bar{\Delta}(q)$ as

$$
\bar{\Delta}(q)=\Delta(q) \bigcup \Delta^{1}(q) \bigcup \cdots
$$

\section{Main Result}

Before stating and proving the paper's main result, we describe the underlying intuition. Fig 2 shows the local geometry of the state space in the vicinity of a point $q_{*}$ on a switching boundary. The shaded cone represents $\gamma_{i}$, the set of possible control vector field selections that might occur when $u_{i}$ is activated. In particular, if $\gamma_{i}\left(q_{*}\right) \bigcap T N_{1}\left(q_{*}\right)=0$, activating $u_{1}$ will ensure that the trajectory of $\Sigma$ can escape $N_{1}$ for any selection in $\gamma_{i}\left(q_{*}\right)$. Then one can apply the classical Chow's theorem to get local controllability. Our goal is to apply the preceding idea to the case where $q_{*}$ lies at the intersection of an arbitrary, but countable, number of switching boundaries. 


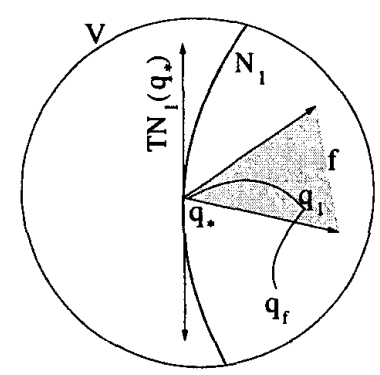

Figure 2: Neighborhood of boundary submanifold.

Theorem 3.1 Let $\left\{N_{k}\right\} \subset \mathbb{R}^{n}$ be a countable set of $\mathcal{C}^{1}$ submanifolds, $\Sigma$ be a control system as in Definition 1.1 where the governing equations are determined by crossing submanifolds in $\left\{N_{k}\right\}$, and $q_{*}$ be a point such that $\bar{\Delta}\left(q_{*}\right)=\mathbb{R}^{n}$. Then $\Sigma$ is small time locally controllable at $q_{*}$

Proof: We proceed by recursion on $p_{q_{*}}$, the number of submanifolds of $\left\{N_{k}\right\}$ intersecting at $q_{*}$. Assume $V$ is an open subset of $\mathbb{R}^{n}, q_{0}, q_{f} \in V$, and $T>0$. Moreover, assume that $\bar{\Delta}\left(q_{*}\right)=\mathbb{R}^{n}$.

First, let $p_{q_{*}}=0$. That is, assume that $q_{*}$ does not lie in any submanifold of the set $\left\{N_{k}\right\}$. Then all the $f_{i}$ in Definition 1.1 are single valued, and $[\cdot, \cdot]$ is therefore single valued, and the classical Chow's theorem holds as in A.1. Therefore the system is small time locally controllable.

For purposes of clarity, before going on to the recursion step, let $p_{q_{*}}=1$ (i.e., $q_{*} \in N_{i}$ for some $i$ ). Order the indices of the $\left\{N_{k}\right\}$ so that $q_{*} \in N_{1}$. Now $\bar{\Delta}=\mathbb{R}^{n}$ implies that there exists $\gamma_{i}$ such that $\gamma_{i} \cap T N_{1}=0$ (if $\gamma_{i} \cap T N_{1} \neq 0$ for all $i$, then elements of $T N_{1}$ are common to all $\gamma_{i}$, implying by Definition 2.2 that $\bar{\Delta}\left(q_{*}\right)$ does not span $\left.\mathbb{R}^{n}\right)$. The condition $\gamma_{i} \cap T N_{1}=0$ implies that there exists $u_{i}:\left[0, \frac{T}{2}\right] \rightarrow \mathbb{R}^{n}$ such that $q(0)=q_{0}, q\left(\frac{T}{2}\right)=q_{1}$ where $q_{1} \in V / N_{1}$ (i.e. input $u_{i}$ will move the system off of $N_{1}$ to some point $q_{1}$ not on $N_{1}$ regardless of the selection from $\gamma_{i}$ - this in turn implies that the flow has reached the interior of a region where the system is single-valued and smooth). Now, by Theorem A.1 $\exists u_{i}^{0}:\left[\frac{T}{2}, T\right] \rightarrow \mathbb{R}^{n}$ such that $q\left(\frac{T}{2}\right)=q_{1}, q(T)=q_{f}$. This implies that the choice of

$$
u_{i}= \begin{cases}u_{i}^{1}, & \text { if } 0<t<\frac{T}{2} \\ u_{i}^{0}, & \frac{T}{2}<t \leq T\end{cases}
$$

satisfies the condition $q(0)=q_{*}, q(T)=q_{f} \forall q_{f} \in V$.

Intuitively, it seems that as $p_{q_{*}} \rightarrow \infty$ it will be more and more difficult for $\Sigma$ to be controllable. This difficulty, however, is embedded in the definition of $\bar{\Delta}$, for if there exists a selection restricting the flow of $\Sigma$ to a submanifold, then by definition $\bar{\Delta}$ does not span $\mathbb{R}^{n}$.

Now assume that for some $k$ the above proposition holds. Then for $k+1$ sub-manifolds intersecting at $q_{*}$, if $\bar{\Delta}=\mathbb{R}^{n}$ then there exists $\gamma_{i}$ such that $\gamma_{i} \cap T N_{k+1}=0$. Therefore, as before, there exists $u_{i}^{k+1}:\left[0, \frac{T}{k}\right] \rightarrow \mathbb{R}^{n}$ such that $q(0)=q_{0}$,

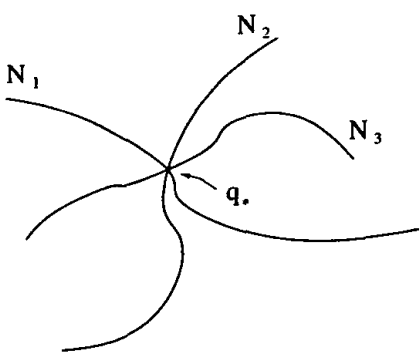

Figure 3: As an example of the proof methodology, $q_{*}$ lies at the intersection of $N_{1}, N_{2}, N_{3} \cdot \bar{\Delta}=\mathbb{R}^{n}$ guarantees that the system trajectory can at least be made to move away from the intersection $N_{1} \cap N_{2} \cap N_{3}$.

$q\left(\frac{T}{k}\right)=q_{k}$ where $q_{k} \in V / N_{k+1}$. Now, by assumptions on the case $k$ there exists $u_{i}^{k}:\left[\frac{T}{k}, T\right] \rightarrow \mathbb{R}^{n}$ such that $q\left(\frac{T}{k}\right)=$ $q_{k}, q(T)=q_{f}$, where

$$
u_{i}^{k}= \begin{cases}u_{i}^{k}, & \text { if } \frac{T}{k+1}<t<\frac{2 T}{k+1} \\ \vdots & \\ u_{i}^{0}, & \frac{k T}{k+1}<t \leq T\end{cases}
$$

This implies that

$$
u_{k}= \begin{cases}u_{i}^{k+1}, & \text { if } 0<t<\frac{T}{k+1} \\ \vdots & \\ u_{i}^{0}, & \frac{k T}{k+1}<t \leq T\end{cases}
$$

satisfies the condition $q(0)=q_{*}, q(T)=q_{f}$. It is therefore true for all $k>0$.

Figure 4 shows an interpretation of Theorem 3.1 for the simple case of the planar system:

$$
\dot{q}=\gamma_{1} u_{1}+\gamma_{2} u_{2}
$$

with $\gamma_{1}$ and $\gamma_{2}$ constant. If $\gamma_{1} \cap \gamma_{2} \neq 0$, the system is not controllable because there exists a selection of $\gamma_{1}$ and a selection of $\gamma_{2}$ which are colinear, thereby restricting the possible evolution of the system to a submanifold. Else, if $\gamma_{1} \cap \gamma_{2}=0$, then the system is controllable because no possible selections from $\gamma_{1}$ and $\gamma_{2}$ will locally restrict the system's flow to a submanifold.
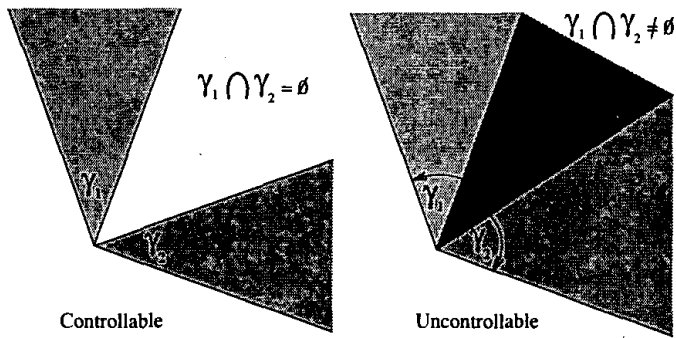

Figure 4: Controllability vs. Noncontrollability

An advantage of this approach is the geometric simplicity of the controllability condition. On the other hand, we are restricted to the assumption that the sub-manifolds $\left\{N_{k}\right\}$ 
determine the governing equations. Failure of our controllability test does not imply that the system is not controllable, only that it is not locally controllable. However, in the cases of interest, such as the example of Section 4, this is acceptable.

\section{Example}

Here we apply the result of Section 3 to a simple example of a three axle vehicle moving in the plane (see Fig. 4). This overconstrained wheeled vehicle is a simplified model of the six-wheeled rocker-bogey mobility system of the Sojourner vehicle that landed on Mars in 1997. This chassis geometry will also be the basis for near-term Mars rover missions. In this example, the front wheel is driven, the middle and back wheels are passive, and the front wheel is always assumed to be in contact with the ground.

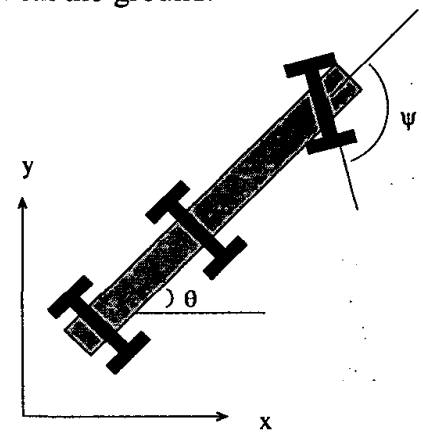

This system is overconstrained, in that its motion can not be determined directly from kinematic constraints (i.e., it's governing equations of motion can not be put in the form of Eq. (A.1)). Except when the vehicle moves straight ahead, at least one of the nonpowered wheels must be slipping at all times. Hence, classical nonholonomic control theories do not apply to this vehicle. In Murphey and Burdick [2000] we proposed a power dissipation method for determining the governing equations of such overconstrained systems when they are moving slowly. The power dissipation function measures the object's total energy dissipation due to contact slippage.

Definition 4.1 The Dissipation or Friction Functional for an $n$-contact state is defined to be

$$
\mathcal{D}=\sum_{i=1}^{n} \alpha_{i}\left|\omega^{i}(q) \dot{q}\right|
$$

where $\alpha_{i}=\mu_{i} F_{i}$, with $\mu_{i}$ and $F_{i}$ being the Coulomb friction coefficient and normal force at the $i^{\text {th }}$ wheel/ground contact, which are assumed known. $\omega^{i}(q) \dot{q}$ represents the velocity of the $i^{\text {th }}$ wheel's point of contact with the ground.

Since one or more of the contact points must always be in a slipping state due to the overconstrained geometry, the power dissipation approach states that the vehicle's motion at any instant is the one that minimizes $\mathcal{D}$, the power lost to slip. In Murphey and Burdick [2000] we showed that the minimum of power dissipation function yields governing equations that are MMDA systems (Definition 1.1).

Using the power dissipation approach, one can show that the minimum of $\mathcal{D}$ must occur when either the middle or back wheel slips. If the vehicle configuration is $q=[x, y, \theta]^{T}$ and the controls $u_{1}$ and $u_{2}$ are associated with the drive and steering velocities respectively, the vehicle's governing equations of motion are:

$$
\begin{aligned}
& \dot{q}=g_{\sigma_{1}}(q) u_{1}+g_{3}(q) u_{2} \quad \sigma_{1}:(q, t) \rightarrow\{a, b\} \\
& g_{1 a}=\left[\begin{array}{llll}
\cos (\psi) \cos (\theta) & \cos (\psi) \sin (\theta) & \frac{1}{l} \sin (\psi) & 0
\end{array}\right]^{T} \\
& g_{1 b}=\left[\begin{array}{c}
\cos (\theta) \cos (\psi)-\frac{r \sin (\theta) \sin (\psi)}{l+r} \\
\cos (\psi) \sin (\theta)+\frac{r \cos (\theta) \sin (\psi)}{l+r} \\
\frac{1}{l+r} \sin (\psi) \\
0
\end{array}\right] \\
& g_{3}=\left[\begin{array}{llll}
0 & 0 & 0 & 1
\end{array}\right]^{T}
\end{aligned}
$$

The function which determines the switching boundaries is:

$$
\Psi(q)=\left(\frac{F_{1} \mu_{1}}{F_{2} \mu_{2}}\right)^{2}\left(\frac{l-r}{r}\right)^{2}-1
$$

where $F_{i}$ are the normal forces above the middle axis and back axis. When $\Psi(g)>0, \sigma_{1}=a$; when $\Psi(g)<0$, $\sigma_{1}=b$. Therefore the switching submanifold $N$ is determined by $\Psi=0$. Intuitively, variations in tire-ground friction and vehicle weight distribution can cause alternations in the choice of the slipping wheel.

Controllability is determined by the rank of the distribution:

$$
\left(g_{3}, g_{\sigma_{1}},\left[g_{3}, g_{\sigma_{1}}\right],\left[\left[g_{3}, g_{\sigma_{1}}\right], g_{\sigma_{1}}\right]\right)
$$

Computing accordingly, we get $\left[g_{\sigma_{1}}, g_{3}\right]=$

$$
\operatorname{co}\left\{\left[\begin{array}{c}
\cos (\theta) \sin (\psi) \\
\sin (\theta) \sin (\psi) \\
\frac{-\cos (\psi)}{l} \\
0
\end{array}\right],\left[\begin{array}{c}
\frac{r \cos (\psi) \sin (\theta)}{l+r}+\cos (\theta) \sin (\psi) \\
\frac{-r \cos (\theta) \cos (\psi)}{l+r}+\sin (\theta) \sin (\psi) \\
-\left(\frac{\cos (\psi)}{l+r}\right) \\
0
\end{array}\right]\right\}
$$

and

$$
\left[g_{\sigma_{1}},\left[g_{\sigma_{1}}, g_{3}\right]\right]=\operatorname{co}\left\{\left[\begin{array}{c}
\frac{-1}{1} \sin (\theta) \\
\frac{1}{l} \cos (\theta) \\
0 \\
0
\end{array}\right],\left[\begin{array}{c}
\frac{-1}{l+r} \sin (\theta) \\
\frac{1}{l+r} \cos (\theta) \\
0 \\
0
\end{array}\right]\right\}
$$

Now, substituting in the algebraic equivalent for $\operatorname{co}\{\cdot, \cdot\}$ we can evaluate the determinant of

$\left[g_{3}, \operatorname{co}\left\{g_{1}, g_{2}\right\},\left[g_{3}, \operatorname{co}\left\{g_{1}, g_{2}\right\}\right],\left[\left[g_{3}, \operatorname{co}\left\{g_{1}, g_{2}\right\}\right], \operatorname{co}\left\{g_{1}, g_{2}\right\}\right]\right]$ $=\left[g_{3}, \operatorname{co}\left\{g_{1}, g_{2}\right\}, \operatorname{co}\left\{\left[g_{3}, g_{1}\right],\left[g_{3}, g_{2}\right]\right\}, \operatorname{co}\left\{\left[g_{1},\left[g_{1}, g_{3}\right]\right],\left[g_{2},\left[g_{2}, g_{3}\right]\right]\right\}\right]$

$=\left[g_{3}, \delta_{1} g_{1}+\left(1-\delta_{1}\right) g_{2}, \delta_{2}\left[g_{3}, g_{1}\right]+\left(1-\delta_{2}\right)\left[g_{3}, g_{2}\right]\right.$, $\left.\delta_{3}\left[g_{1},\left[g_{1}, g_{3}\right]\right]+\left(1-\delta_{3}\right)\left[g_{2},\left[g_{2}, g_{3}\right]\right]\right]$

where $\delta_{i} \in[0,1]$ for $i=1,2,3$. The determinant is

$$
\frac{\left(l+r \delta_{3}\right)\left(2 l+r\left(\delta_{1}+\delta_{2}\right)+r\left(\delta_{2}-\delta_{1}\right) \cos (2 \psi)\right)}{2 l^{2}(l+r)^{2}}
$$


which equals 0 only if $\delta_{3}<0$ which is not an admissible $\delta_{3}$. Hence, the vehicle is always STLC, as expected. Physically, this result implies that the vehicle remains locally controllable even as the status of the slipping wheel alters unexpectedly.

\section{Conclusions}

Understanding the issue of controllability is often a first step toward understanding how to control a class of nonlinear systems. This paper studied multiple model systems where the individual plants are driftless affine, and the plant selection is determined by submanifolds in the state space. We extended Chow's classical theorem to this class of control systems. Because of the potential importance of the vehicles discussed in Section 4, future work will investigate algorithms for stabilizing the multi-model systems of Definition 1.1. Preliminary methods for open loop motion planning of such systems can be found in Murphey and Burdick [2001]. We also desire to extend the current results to more general cases where switching between models is not determined solely by submanifold boundaries. Such a result will almost certainly depend on developing an appropriate open mapping theorem for differential inclusions, probably similar to that found in Sussman [1998].

Acknowledgements: This work was partially supported by the National Science Foundation (grant NSF9402726) through its Engineering Research Center (ERC) program.

\section{References}

E. Asarin, O. Bournez, T. Dang, O. Maler, and A. Pneuli. Effective synthesis of switching controllers for linear systems. Proc. IEEE, 88(7):1011-1025, July 2000.

Michael S. Branicky. Multiple lyapunov functions and other analysis tools for switched and hybrid systems. IEEE Transactions on Automatic Control, 43(4):475-482, April 1998.

W.L. Chow. Über systeme von linearen partiellen differentialgeichungen erster ordinung. Math Ann., 117:98-105, 1939.

W.P. Dayawansa and C.F. Martin. A converse lyapunov theorem for a class of dynamical systems which undergo switching. IEEE Trans. Automatic Control, 44(4):751-760, Apr. 1999.

A.F. Filippov. Differential Equations with Discontinuous Right Hand Sides. Kluwer Academic Publishers, 1988

Bill Goodwine and Joel W. Burdick. Controllability of kinematic control systems on stratificd configuration spaces. IEEE Trans. on Automatic Control, 46(3):358-368, 2000.

J. P. Hespanha, D. Liberzon, and A. S. Morse. Logic-based switching control of a nonholonomic system with parametric uncertainty. Systems Control Lett., 38:167-177, 1999.

Joao Hespanha, Daniel Liberzon, A. Stephen Morse, Brian D. O. Anderson, Thomas S. Brinsmead, and Franky De Bruyne. Multiple model adaptive control, part 2: Switching. Int. J. of Robust and Nonlinear Control Special Issue on Hybrid Systems in Control, 11(5):479-496, April 2001.

Daniel Liberzon and A. Stephan Morse. Basic problems in stability and design of switched systems. IEEE Control System Mag., 19 (5):59-70, 1999.
T. D. Murphey and J. W. Burdick. Issues in controllability and motion planning for overconstrained wheeled vehicles. In Proc. Int. Conf. Math. Theory Networks Systems (MTNS), Perpignan, France, 2000.

T. D. Murphey and J. W. Burdick. A controllability test and motion planning primitives for overconstrained vehicles. In Proc. IEEE Int. Conf. on Robotics and Automation, Seoul, Korea, 2001.

G.J. Pappas, G. Laffierier, and S. Sastry. Hierarchically consistent control sytems. IEEE Trans. Automatic Control, 45(6):1144 1160 , June 2000.

Franco Rampazzo and Hector J. Sussman. Set-valued differentials and a nonsmooth version of chow's theorem. In (preprint) submitted to 40th Conf. Decision Control, 2001

Hector Sussman. Multidifferential calculus: Chain rule, open mapping and transversal intersection theorems. In Optimal Control: Theory, Algorithms, and Applications (proceedings of the 1997 IFIP Conf.), pages 436-487. Kluwer Academic Publishers, 1998.

M. Zefran and J.W. Burdick. Design of switching controllers for systems with changing dynamics. In Workshop on Hybrid Systems, Computation and Control, Berkeley, CA, 1998.

\section{A Review of Chow's Theorem}

For convenience, we review the classical theorem of Chow [1939]. Let $Q$ be an $n$-dimensional manifold, $T Q$ its tangent bundle, and $\mathcal{U}$ the $m$-dimensional space of inputs. Drift free affine control systems take the form:

$$
\dot{q}=g_{1}(q) u_{1}+g_{2}(q) u_{2}+\cdots+g_{m}(q) u_{m}
$$

where $q \in Q,\left(u_{1}, \ldots, u_{m}\right)$ are the controls, and $g_{1}, \ldots, g_{m}$ are smotth control vector fields. Intuitively, small time local controllability (STLC) implies that admissible controls can be found to locally steer the system from a given starting point to any configuration in a neighborhood of that point. Formally,

Definition A.1 (STLC) A drift free control system of the form Eq. (A.1) is small time locally controllable (STLC) if given an open subset $V \subset \mathbb{R}^{n}$ and $q_{0}, q_{f} \in V$ and $T>0$, there exists $u:[0, T] \rightarrow \mathcal{U}$ such that $q(0)=q_{0}$ and $q(T)=q_{f}$ with $q(t) \in V$ for all $t \in[0, T]$.

In coordinates, the Lie Bracket $[f, g]$ between two vector fields $f$ and $g$ is

$$
[f, g](q)=\frac{\partial g}{\partial q} f(q)-\frac{\partial f}{\partial q} g(q)
$$

The distribution $\Delta$ of the control vector fields in (A.1) is the span of those vector fields, and the involutive closure, $\bar{\Delta}$, of $\Delta$ is the closure of $\Delta$ under the Lie bracketing operation. Using these operations, we state the classical result of Chow [1939].

Theorem A.1 (Chow) The control system (A.1) is small time locally controllable at $q \in \mathbb{R}^{n}$ if $\bar{\Delta}_{q}=T_{q} \mathbb{R}^{n}$. 